\title{
Importance of metabolomics analyses of maternal parameters and their influence on fetal growth (Review)
}

\author{
JINFENG LIU*, GANG LIU* ${ }^{*}$ and ZHENGUANG LI \\ Department of Neonatology, Xuzhou Children's Hospital, Xuzhou, Jiangsu 221002, P.R. China
}

Received February 15, 2017; Accepted May 18, 2017

DOI: $10.3892 /$ etm.2017.4517

\begin{abstract}
Metabolomics is the scientific study of chemical processes involving metabolites. Specifically, metabolomics is the systematic study of the unique chemical fingerprints that specifically conveys cell processes. Fetal growth aberrations, including fetal growth restriction and macrosomia, convey the highest risk of perinatal mortality and morbidity, as well as increasing the chance of developing chronic disease in later life. We searched the electronic database PubMed for preclinical as well as clinical controlled studies pertaining to metabolomics analyses of maternal parameters and their influence on fetal growth. It was observed clearly that metabolic profiling/metabolomics approaches in maternal urine samples provide information on early-life exposure and are potentially linked to child health outcomes, in addition to identifying new biomarkers of exposure. This review article is aimed to discuss intra- and inter-individual variations in maternal urine profiles during pregnancy, fetal growth outcomes and environmental sources of metabolic variations. The review concludes that metabolic profiling of mother is a useful tool for the evaluation of influences on the growth of the fetus.
\end{abstract}

\section{Contents}

1. Introduction

2. Fetal growth outcomes: Definitions and tools for characterization

3. Environmental determinants of abnormal fetal growth and long-term consequences

4. Metabolic profiling

5. Applications of metabolic profiling

6. Metabonomics application in epidemiology of chronic diseases

7. Conclusion

Correspondence to: Dr Zhenguang Li, Department of Neonatology, Xuzhou Children's Hospital, 18 Sudibei Road, Xuzhou, Jiangsu 221002, P.R. China

E-mail: zhenguangli29@163.com

*Contributed equally

Key words: maternal parameters, growth, child health

\section{Introduction}

The human development timetable is unique compared to other primates (1). Late brain development is necessary for humans in order to house a large skull and forebrain in adulthood while maintaining a narrow pelvic birth canal required for an upright posture for walking (2). From the gametes to the embryo and functioning placenta, the information required to develop from a single fertilized cell (zygote) to an autonomous human being is contained in the 35,000 genes of this initial cell. During development, the genetic information is unfolded to control morphologic development. This set of genes consists of chromosomes half of which are from the mother and the remaing half from the father. These chromosomes are combined during the fertilization stage, which is defined as the time when the spermatozoid and the secondary oocyte are in contact. Thirty hours after fertilization the zygote undergoes a series of cleavage divisions maintaining the same total mass (3). Further cellular division is accompanied by the formation of a small cavity between the cells, forming the blastocyst. In the next days the blastocyst, which is at this stage only a ball of identical cells, starts to develop a polarity and different compartments are formed. The inner cell mass forms the embryo and the other components constitute the placenta and other supporting tissues. The embryo must grow out of the conceptus (to 'hatch') to continue development and implants into the uterine lining. By that stage, the blastocyst would have travelled down the fallopian tubes into the uterus.

The placenta formation also requires the component of the blastocyst to grow out, the specialized epithelial cells form the outer layer of the blastocyst, and invade the maternal uterine wall. This process is known as trophoblastic invasion. Once the invasion has occurred the development of the uteroplacental circulation system allows the circulatory system of the mother and of the embryo to exchange gases and nutrients through simple diffusion. At this stage the embryo is exposed to sudden high levels of oxygen and needs to produce antioxidant enzymes. Trophoblastic invasion and/or antioxidant reactions are believed to fail in hypertensive disorders such as gestational hypertension and preeclampsia which are the leading cause of maternal mortality in developed countries, accounting for $16 \%$ of deaths (4). Oxidative stress can also affect the normal placental development and perturb nutrient availability later on during fetal growth. The placenta has a vast range of functions that is described briefly below. Most 
importantly, it allows nutrient exchange between maternal and fetal blood but creates an immune barrier between the fetus and genetically different mother by not allowing direct contact of the two circulatory systems. The placenta acts as the respiratory site for the fetus as well as a site of filtration for plasma nutrients and wastes. This exchange occurs in a way that the fetal blood flows through the umbilical arteries to the placenta, then into finer vessels, capillaries that go through a spongelike material (chorionic villus, analveolus-like structure) where exchange occurs between maternal uterine blood and fetal blood. Replenished fetal blood finally flows into one large umbilical vein that returns to the fetus. The placenta is also the site of hormone secretion that alters maternal physiology and supports fetal growth (3). Placental size can vary greatly between pregnancies adapting to different environments. For example, at high altitude $(>2,500 \mathrm{~m})$ where low oxygen levels are observed, pregnant women present larger placenta relative to fetal size. This is to benefit oxygen diffusion (5). The placenta is the most important determinant of the fetal environment. During the embryonic period which lasts 8 weeks from the moment of implantation, most organ systems are established, such as the heart, the gastrointestinal tract and the liver, and this with great rapidity. Therefore, it is also the most vulnerable time during pregnancy where most toxic exposures to teratogens result in an 'all or none effect' (meaning any effect may be so profound as to cause a spontaneous abortion). By the end of the embryonic period, the incidence of malformations that lead to miscarriages decreases from $>10$ to $1 \%$ (6).

\section{Fetal growth outcomes: Definitions and tools for characte- rization}

Fetal growth restriction (FGR) or intrauterine growth restriction (IUGR) occurs when fetuses fail to achieve their genetically determined weight and size. The ideal weight and size for each individual is complex to determine in clinical settings. In clinical practice, FGR is diagnosed by comparing the birth weight and other length measurements, such as the abdominal or head circumferences at birth and/or during the fetal period (ultrasound measurements), to a population-based growth chart. Small for gestational age (SGA) is generally used as a surrogate for FGR, classically defined as a birth weight $<10$ th percentile, using sex-adjusted centile charts. However, among neonates classified as SGA, only $25 \%$ may be constitutionally small according to different studies which determined constitutional variation due to maternal height, weight, paternal height, ethnic group and parity (7). Customized growth charts were found more likely to detect neonates at risk of perinatal morbidity and mortality than would be detected by a population chart (8). The challenge for obstetricians is to differentiate constitutionally small fetuses to pathologically growth restricted and intervene with induced premature delivery if necessary. The diagnosis of abnormal fetal growth, and possibly prognostic one, would also be better for postnatal care and provide closer clinical monitoring for subsequent pregnancies. However, 40-80\% of FGR cases remain undiagnosed before birth, contributing to high perinatal mortality (9). In epidemiological settings, simpler metrics have been used to study fetal growth determinants such as continuous birth weight or low birth weight (LBW;
$<2,500 \mathrm{~g}$ ) often restricted to the term LBW, which need to be corrected for the gestational age directly or at the stage of the regression modelling with weight determinants by statistical adjustment (10). Besides IUGR, fetal macrosomia, also defined as large for gestational age (LGA), is characterised by a birth weight above the 90th percentile or authors have variably defined it as a birthweight $>4,000,>4,500$ or $>5,000 \mathrm{~g}$ (11).

Common screening strategies for prenatal outcomes include non-invasive tools such as ultrasonography and serum biomarker screening sometimes in combination with invasive methods such as amniocentesis, for women at risk (>35 years old). For example, second-trimester measurements of $\alpha$-fetoprotein, human chorionic gonadotropin, and estriol in maternal blood can help identify $60-70 \%$ of cases of aneuploidy (abnormal number of chromosomes in a cell such as in Down syndrome). Novel tools to assess chromosomic disorders and alteration in placentation may include the analysis of fetal cell material in maternal blood. At the present time, no biomarkers were found clinically useful and urine biomarkers have remained largely unexplored (12).

FGR can be classified into two types: asymmetric, which is the most common type, and symmetric. Asymmetric growth can take place if the fetus is subject to reduced oxygen or nutrient supply during the last trimester of pregnancy due to deficient vascular-placental functions. As a result body weight and sometimes length is reduced but the head circumference is maintained as normal. A symmetrical growth restriction consists of an equally distributed growth impairment of the head, weight and length occurring during early pregnancy (13). FGR can have intrinsic origins stemming from fetal conditions such as genetic abnormalities or congenital infections. However, extrinsic aetiologies account for the majority of FGR cases including maternal and placental conditions. In fact, most of the pregnancy complications are believed to be the clinical manifestations of underlying placental ischemia (restriction of blood supply) and utero-placental deficiency (14). The ischemic placental diseases comprise preeclampsia, FGR, placental abruption and fetal distress. In developing countries FGR is primarily the consequence of low energy intake associated with low BMI and low gestational weight gain. By contrast, in the developed world, FGR is prevalent among women with pregnancy-induced hypertension, smoking habits and poor nutritional status (7). Previous findings emphasised the importance of maternal diet, such as fruit intake and green leafy vegetable intake on the incidence of FGR (15). It was also shown that the consumption of fish and the high adherence to a Mediterranean diet among women in the INMA cohort improved birth weight outcomes (16). By contrast, fetal macrosomia is strongly correlated with high maternal glucose levels and gestational diabetes mellitus (GDM) and often means that the delivery needs to occur through caesarean section.

Advances in estimation of fetal adiposity via ultrasounds also allowed to better distinguish constitutionally to pathologically overgrown babies. Indeed, fetal adiposity, which constitutes only $14 \%$ of birth weight, accounts for half of the weight variation observed across a population (17). Animal studies also demonstrated that fat-free mass at birth shows high consistency across individuals (18). Determinants of birth weight were shown to account for only $30 \%$ of the population variation, and even less for fetal adiposity (17\%) including maternal height, 
pregravid weight, weight gain during pregnancy, education, parity, paternal height and weight, neonatal sex, and gestational age.

\section{Environmental determinants of abnormal fetal growth and long-term consequences}

During prenatal and early postnatal life, i.e., during the time of cell differentiation and organ formation, the organism is highly sensitive to environmental constraints such as nutrients, environmental chemicals, drugs, infections and other stressors. This developmental period of plasticity may allow for adaptation to the changing environments in order to maintain or improve health and reproductive capability (14). However, this environment can also have adverse consequences on later life functions. The fetal origin of adult diseases is believed to play an important role in the progression of non-communicable diseases over the last 40 years such as cardiovascular diseases, obesity and type 2 diabetes, dysfunctions of the reproductive, brain and immune system; although, western lifestyle and genetic background are known to predispose to these diseases (18). However, the genetic component is believed to play only a small role, estimated to explain $20 \%$ of the variation observed in BMI, as based on a recent genome-wide study of 339,224 individuals (19). In utero exposure to toxic chemicals or to poor or overnutrition can result in clinical manifestations, such as death, birth defects and low birth weight, or more subtle functional changes. Exposures to low doses of environmental chemicals during development, especially those with endocrine activity, can result in functional changes in gene expression, that have no phenotype at birth but that lead to increased risk of disease and dysfunction later in life. This knowledge can have an impact on prenatal care, in the attempt to prevent perinatal outcomes, but also on the understanding and management of chronic diseases.

Evidence suggests that a number of environmental chemicals alter disease susceptibility as a result of prenatal exposure. These include bisphenol A (from polycarbonate plastics), phthalates (softener in plastics) some organophosphate and organochlorine pesticides, nicotine (smoking), air pollution, mercury, perfluorooctanoic acids (stain and water repellents), and polybrominated diphenyl ethers (flame retardants), chemicals that are found in detectable concentrations in blood or urine samples in most individuals. Adverse developmental effects from certain pollutants may be small, for instance drinking water contaminants, but the large number of women exposed means that the population attributable risk is potentially high and may be reduced by appropriate water treatment. The identification of environmental risk factors of FGR and early prognostic biomarkers (i.e., before clinical outcome manifests) would greatly improve obstetric counselling and decision making.

\section{Metabolic profiling}

Metabolic profiling has great potential to serve environmental exposure sciences and pregnancy research as a tool to identify intermediate biomarkers linking exposure and outcomes. This original concept was defined previously as the 'meet-in-the-middle' approach where preclinical biomarkers correlated with specific exposures in prospective studies may be elevated in cases and support the causal association between exposure and outcomes (20). Previous findings served as a proof-of-concept where for instance, metabonomic signatures of colon cancer were related to dietary fibre intake in the European Prospective Investigation into Cancer and Nutrition (EPIC) study (21).

\section{Applications of metabolic profiling}

Metabolic profiling has been applied in various clinical settings, particularly recently in prenatal care, as well as in epidemiology of chronic diseases and environmental exposures which are discussed below.

Metabolic profiling applied to neonatal research has mainly emerged as a non-targeted approach, to explore potential biomarkers of reproductive outcomes and identify underlying biological mechanisms. Human studies have focused on maternal plasma, amniotic fluid, urine and cervicovaginal excretions. Metabolic analyses of placenta and cord blood provided further information on the placental nutrient transfer especially of amino acids. Preeclampsia, which is characterized by high blood pressure and proteinuria, has been the main focus in metabolic profiling applications in fetal research. Studies investigating fetal growth outcomes, including IUGR and fetal macrosomia, are sparser (22). A systematic search was conducted looking for studies published in peer-reviewed journals before June 2015 including terms such as 'metabolic profiling', 'metabonomics' 'metabolomics' methods applied in humans during pregnancy only to assess 'fetal growth', 'low birth weight', 'LBW', 'intrauterine growth restriction', 'IUGR', 'birth weight'. Horgan et al produced a comprehensive work applying LC-MS-based metabolic profiling in three different studies including in maternal plasma early in gestation and in venous cord plasma in association with SGA. The authors of that study suggested a multifactorial origin of SGA and a potential for a presymptomatic screening test for SGA (22). The study provided useful insights into potential mechanisms of SGA across species, including general differential change in sphingolipids, phospholipids, carnitines and fatty acids. A general increase of these metabolites was measured in maternal plasma of SGA cases whereas they were decreased in cord blood, demonstrating a failure of the placenta to transfer essential nutrients to the fetus. However, the study size was limited and did not allow controlling for factors known to affect fetal growth such as gestational hypertension and smoking (22). Another study found similar metabolic signatures of poor pregnancy outcomes including SGA, preterm birth and neonatal intensive care admission in maternal serum in third trimester (23). Mothers with poor pregnancy outcomes showed differences in plasma levels during the second trimester of pregnancy in lipids (sterols, glycerophospholipids, sphingolipids), steroid hormones (progesterone metabolites) and vitamin D metabolites compared to controls $(n=40)(23)$. Another recent study using LC-MS on maternal serum in early pregnancy (12-14th weeks) could predict the risk for birthweight $>4000 \mathrm{~g}(\mathrm{n}=20)$ but not LBW $(\mathrm{n}=19)(24)$. Mechanistic insights were provided based on lower levels of phospholipids, lysophospholipids, and monoacylglycerols early in pregnancy in mothers with fetal macrosomia, suggesting an 
increased transport of lipids to the fetus (also supported by an indirect correlation between placental lipid transporters adipocyte fatty acid-binding protein and maternal free lipids). These three studies were biased towards lipid species as a consequence of applying reversed-phase UPLC-MS, where the majority of polar species, including amino acids and organic acids, cannot be detected.

To the best of our knowledge, there is only one study reporting differences in maternal $1 \mathrm{H}$ NMR metabolic profiles from serum/plasma and urine where only 10 IUGR cases were available (25). Another study analysed maternal blood and cord blood samples from very LBW infants by $1 \mathrm{H}$ NMR $(\mathrm{n}=8)$ and found metabolic signature of mothers delivering VLBW infants, including decreased levels of acetate and increased levels of lipids, pyruvate, glutamine, valine and threonine (26). These studies highlighted potential differences in materno-fetal nutrient transfer using cord blood analysis and, probably, short-term changes associated with birth-related oxidative stress. However, these studies showed a lack of consistent classification of SGA/IUGR and focus on a single disease. Indeed, Tea et al (26) compared mothers with VLBW infants to controls where $63 \%$ of the cases had hypertension in contrast to 0 among controls. Besides IUGR, fetal macrosomia, defined as LGA, was also the focus of a study using $1 \mathrm{H}$ NMR on first trimester maternal urine in 50 participants (27). Authors of that study found that urinary metabolic profiles differed significantly according to fetal fat disposition in utero with increased levels of taurine, and histidine.

The principal limitation of those studies is the small population size, with between 5 and 60 subjects per group. This limits the transferability of the results to a general population with different ethnicities and/or diets. Small population size also did not allow targeting a single disease or a specific stage of disease. Indeed, clinical disparity between pregnancy outcomes such as FGR, preterm birth, preeclampsia and gestational diabetes should be recognised in studies applying metabolic profiling because of their different aetiologies (28). In addition, small sample size, prevented control for crucial confounding factors such as maternal smoking or maternal hypertension. In a recent study, we demonstrated the value of urine metabolomics in detecting early markers (at approximately 11 weeks) of preterm birth and FGR in a large nested case-control study with $>400$ participants (29). Metabolomics was proven useful also in neonatal medicine, although in small studies, with the ability to further understanding of prenatal life, IUGR outcomes and subsequent development of metabolic diseases.

\section{Metabonomics application in epidemiology of chronic diseases}

Genetic programming or single environmental exposure cannot only explain complexity in chronic disease risk factors. Most chronic diseases are the results of gene-environment interactions operating at a population level and at the individual level. Application of metabonomics to investigate the source of phenotypic variations and chronic disease risk factors in large scale cohorts allow invenstigators to simultaneously capture these levels of complexity. Metabolome-wide association studies (MWASs) have emerged as top-down approaches where metabolic phenotypes (the endpoint of physiological changes) are used to explore systematic statistical associations with disease risk factors, in order to generate hypotheses on disease aetiology and further test them in more controlled systems (cell assays, animal models or interventional studies) (30). Concerns were raised on the clinically predictive potential of metabolic profiles because of its high temporal variability. However, it was shown that $60 \%$ (plasma) and $47 \%$ (urine) of biological variation in 1H NMR-detectable metabolite concentrations was stable and representative of familial and individual environmental factors (31). Other recent studies confirmed that the stable component of inter-individual variation or intraclass correlation coefficient, over a 4-month to a year interval was between 0.43 and 0.57 (32).

Modern epidemiological studies often include the collection of blood and urine samples, which are rich in a wide range of metabolites such as lipids, amino acids, small proteins, bile acids and other intermediate metabolites. Blood specimens are a 'snapshot' of the global metabolism at the time of collection but can offer limited information on inter-individual metabolic variation since its metabolite content is under tight homeostatic control $(4,6)$. In comparison, urine collection is non-invasive and more cost-effective and might be preferred in certain population (e.g., mother-child cohort). Urine specimens supply a time-averaged view of the global metabolism including end-products of endogenous and exogenous metabolism. Additional non-invasive biological samples may be available such as hair, nails, saliva or placenta in prospective studies although their collection, storage and analytical procedures are less established when compared to urine and blood (32).

Metabolic profiling and multivariate statistical modelling approaches have successfully been applied to large-scale epidemiological studies of chronic diseases, for example, in the MWAS of the INTERMAP cohort (33). This prospective study assessed the association between blood pressure and lifestyle factors by obtaining the metabolic phenotype of 4,630 individuals in 17 different population samples from China, Japan, United Kingdom and the United States. Over 10 biomarkers were identified for blood pressure, related to diet, gut microbiota, drug use and host genetic influences. More recently, urinary metabolic signatures of BMI were found in the INTERMAP study (34). Metabolic profiles (LC-MS) could also predict the development of type 2 diabetes as shown in the Framingham Heart Study (35). A combination of three amino acids gave a $>5$-fold higher risk for individuals in the top quartile, which included the branched-chain and aromatic amino acids isoleucine, leucine, valine, tyrosine, and phenylalanine. These findings were validated in multiple studies subsequently (36).

Metabolic profiling applications were also proven useful in nutritional epidemiology. This approach often strengthens traditional self-reported dietary assessment methods that are subject to recall bias and measurement error and can offer mechanistic insights (37). From a public health perspective metabonomics can help in identifying modifiable risk factors that contribute to changes in metabolite concentrations related to disease risk. Convincing evidence are coming from reports based on the EPIC study conducted in 10 European countries to investigate the relationship between diet and risk of cancer. Meat and butter intake were both found as direct biomarkers 
related to colon cancer risk (38). However, the mean proportion of variation of serum metabolites (by LC-MS; Biocrates) that was explained by habitual diet was small, ranging from $3.6 \%$ for amino acids to $7.7 \%$ for a choline-containing phospholipid. Interventional nutritional studies offered great validation using metabonomics to find intermediate pathways explaining dietary-related cancer risks. This is best shown by the recent 2-week food exchange study in subjects from the same populations, where individuals of African American descent were fed a high-fibre, low-fat African-style diet and those of rural African descent a high-fat, low-fibre western-style diet (39). The food changes resulted in notable reciprocal changes in mucosal biomarkers of colon cancer risk and offered insights in aspects of the microbiota and metabolome known to affect cancer risk.

\section{Conclusion}

Metabolic profiling in the context of general human population, in contrast to controlled animal studies, can offer great insights into the actual impact of the environment and lifestyle on disease risks taking into account genetic and medical history diversity. Several limitations were identified in the literature in particular the presence of false-positive findings in association studies that use data derived from high-throughput metabolomics technologies.

\section{References}

1. Posobiec LM, Cox EM, Solomon HM, Lewis EM, Wang KF and Stanislaus D: A probability analysis of historical pregnancy and fetal data from Dutch Belted and New Zealand White Rabbit Strains from embryo-fetal development studies. Birth Defects Res B Dev Reprod Toxicol 107: 76-84, 2016.

2. Falk D, Zollikofer CPE, Morimoto N and Ponce de León MS: Metopic suture of Taung (Australopithecus africanus) and its implications for hominin brain evolution. Proc Natl Acad Sci USA 109: 8467-8470, 2012

3. Niakan KK, Han J, Pedersen RA, Simon C and Pera RA: Human pre-implantation embryo development. Development 139: 829-841, 2012.

4. Khan KS, Wojdyla D, Say L, Gülmezoglu AM and Van Look PF: WHO analysis of causes of maternal death: a systematic review. Lancet 367: 1066-1074, 2006.

5. Moore LG, Charles SM and Julian CG: Humans at high altitude: hypoxia and fetal growth. Respir Physiol Neurobiol 178: 181-190, 2011.

6. Wilcox AJ, Weinberg CR, O'Connor JF, Baird DD, Schlatterer JP, Canfield RE, Armstrong EG and Nisula BC: Incidence of early loss of pregnancy. N Engl J Med 319: 189-194, 1988.

7. McCowan L and Horgan RP: Risk factors for small for gestational age infants. Best Pract Res Clin Obstet Gynaecol 23 779-793, 2009

8. McCowan LM, Harding JE and Stewart AW: Customized birthweight centiles predict SGA pregnancies with perinatal morbidity. BJOG 112: 1026-1033, 2005.

9. Chang TC, Robson SC, Boys RJ and Spencer JA: Prediction of the small for gestational age infant: which ultrasonic measurement is best? Obstet Gynecol 80: 1030-1038, 1992.

10. Slama R, Ballester F, Casas M, Cordier S, Eggesbø M, Iniguez C Nieuwenhuijsen M, Philippat C, Rey S, Vandentorren S, et al Epidemiologic tools to study the influence of environmental factors on fecundity and pregnancy-related outcomes. Epidemiol Rev 36: 148-164, 2014.

11. Zhang X, Decker A, Platt RW and Kramer MS: How big is too big? The perinatal consequences of fetal macrosomia. Am J Obstet Gynecol 198: 517.e1-6, 2008.

12. Conde-Agudelo A, Papageorghiou AT, Kennedy SH and Villar J Novel biomarkers for predicting intrauterine growth restriction: a systematic review and meta-analysis. BJOG 120: 681-694, 2013.
13. Wollmann HA: Intrauterine growth restriction: definition and etiology. Horm Res 49 (Suppl 2): 1-6, 1998.

14. Ananth CV and Vintzileos AM: Maternal-fetal conditions necessitating a medical intervention resulting in preterm birth. Am J Obstet Gynecol 195: 1557-1563, 2006.

15. Mitchell EA, Robinson E, Clark PM, Becroft DM, Glavish N, Pattison NS, Pryor JE, Thompson JM and Wild CJ: Maternal nutritional risk factors for small for gestational age babies in a developed country: a case-control study. Arch Dis Child Fetal Neonatal Ed 89: F431-F435, 2004.

16. Chatzi L, Mendez M, Garcia R, Roumeliotaki T, Ibarluzea J, Tardón A, Amiano P, Lertxundi A, Iñiguez C, Vioque J, Kogevinas M, Sunyer J; INMA and RHEA study groups: Mediterranean diet adherence during pregnancy and fetal growth: INMA (Spain) and RHEA (Greece) mother-child cohort studies. Br J Nutr 107: 135-145, 2012.

17. Catalano PM, Tyzbir ED, Allen SR, McBean JH and McAuliffe TL: Evaluation of fetal growth by estimation of neonatal body composition. Obstet Gynecol 79: 46-50, 1992.

18. Catalano PM and Kirwan JP: Maternal factors that determine neonatal size and body fat. Curr Diab Rep 1: 71-77, 2001.

19. Locke AE, Kahali B, Berndt SI, Justice AE, Pers TH, Day FR, Powell C, Vedantam S, Buchkovich ML, Yang J, et al; LifeLines Cohort Study; ADIPOGen Consortium; AGEN-BMI Working Group; CARDIOGR AMplusC4D Consortium; CKDGen Consortium; GLGC; ICBP; MAGIC Investigators; MuTHER Consortium; MIGen Consortium; PAGE Consortium; ReproGen Consortium; GENIE Consortium; International Endogene Consortium: Genetic studies of body mass index yield new insights for obesity biology. Nature 518: 197-206, 2015.

20. Vineis P and Perera F: Molecular epidemiology and biomarkers in etiologic cancer research: the new in light of the old. Cancer Epidemiol Biomarkers Prev 16: 1954-1965, 2007.

21. Chadeau-Hyam M, Athersuch TJ, Keun HC, De Iorio M, Ebbels TM, Jenab M, Sacerdote C, Bruce SJ, Holmes E and Vineis P: Meeting-in-the-middle using metabolic profiling - a strategy for the identification of intermediate biomarkers in cohort studies. Biomarkers 16: 83-88, 2011.

22. Horgan RP, Broadhurst DI, Walsh SK, Dunn WB, Brown M, Roberts CT, North RA, McCowan LM, Kell DB, Baker PN, et al: Metabolic profiling uncovers a phenotypic signature of small for gestational age in early pregnancy. J Proteome Res 10: 3660-3673, 2011.

23. Heazell AE, Bernatavicius G, Warrander L, Brown MC and Dunn WB: A metabolomic approach identifies differences in maternal serum in third trimester pregnancies that end in poor perinatal outcome. Reprod Sci 19: 863-875, 2012.

24. Ciborowski M,Zbucka-Kretowska M, Bomba-Opon D, Wielgos M, Brawura-Biskupski-Samaha R, Pierzynski P, Szmitkowski M, Wolczynski S, Lipinska D, Citko A, et al: Potential first trimester metabolomic biomarkers of abnormal birth weight in healthy pregnancies. Prenat Diagn 34: 870-877, 2014.

25. Diaz SO, Barros AS, Goodfellow BJ, Duarte IF, Galhano E, Pita C, Almeida MC, Carreira IM and Gil AM: Second trimester maternal urine for the diagnosis of trisomy 21 and prediction of poor pregnancy outcomes. J Proteome Res 12: 2946-2957, 2013.

26. Tea I, Le Gall G, Küster A, Guignard N, Alexandre-Gouabau MC, Darmaun D and Robins RJ: 1H-NMR-based metabolic profiling of maternal and umbilical cord blood indicates altered maternofoetal nutrient exchange in preterm infants. PLoS One 7: e29947, 2012.

27. Walsh JM, Wallace M, Brennan L and McAuliffe FM: Early pregnancy maternal urinary metabolomic profile and later insulin resistance and fetal adiposity. J Matern Fetal Neonatal Med 28: 1697-1700, 2015.

28. Fanos V, Atzori L, Makarenko K, Melis GB and Ferrazzi E: Metabolomics application in maternal-fetal medicine. BioMed Res Int 2013: 720514, 2013

29. Maitre L, Fthenou E, Athersuch T, Coen M, Toledano MB, Holmes E, Kogevinas M, Chatzi L and Keun HC: Urinary metabolic profiles in early pregnancy are associated with preterm birth and fetal growth restriction in the Rhea mother-child cohort study. BMC Med 12: 110, 2014

30. Nicholson JK, Holmes E and Elliott P: The metabolome-wide association study: a new look at human disease risk factors. J Proteome Res 7: 3637-3638, 2008.

31. Nicholson G, Rantalainen M, Maher AD, Li JV, Malmodin D, Ahmadi KR, Faber JH, Hallgrímsdóttir IB, Barrett A, Toft $\mathrm{H}$, et al; The MolPAGE Consortium: Human metabolic profiles are stably controlled by genetic and environmental variation. Mol Syst Biol 7: 525-525, 2011. 
32. Sampson JN, Boca SM, Shu XO, Stolzenberg-Solomon RZ, Matthews CE, Hsing AW, Tan YT, Ji BT, Chow WH, Cai Q, et al: Metabolomics in epidemiology: sources of variability in metabolite measurements and implications. Cancer Epidemiol Biomarkers Prev 22: 631-640, 2013.

33. Holmes E, Loo RL, Stamler J, Bictash M, Yap IK, Chan Q, Ebbels T, De Iorio M, Brown IJ, Veselkov KA, et al: Human metabolic phenotype diversity and its association with diet and blood pressure. Nature 453: 396-400, 2008.

34. Elliott P, Posma JM, Chan Q, Garcia-Perez I, Wijeyesekera A, Bictash M, Ebbels TM, Ueshima H, Zhao L, van Horn L, et al: Urinary metabolic signatures of human adiposity. Sci Transl Med 7: 285ra62, 2015

35. Wang TJ, Larson MG, Vasan RS, Cheng S, Rhee EP, McCabe E, Lewis GD, Fox CS, Jacques PF, Fernandez C, et al: Metabolite profiles and the risk of developing diabetes. Nat Med 17: 448-453, 2011.
36. Adams SH: Emerging perspectives on essential amino acid metabolism in obesity and the insulin-resistant state. Adv Nutr 2: 445-456, 2011.

37. Guertin KA, Moore SC, Sampson JN, Huang WY, Xiao Q, Stolzenberg-Solomon RZ, Sinha R and Cross AJ: Metabolomics in nutritional epidemiology: identifying metabolites associated with diet and quantifying their potential to uncover diet-disease relations in populations Am J Clin Nutr 100: 208-217, 2014.

38. Floegel A, von Ruesten A, Drogan D, Schulze MB, Prehn C, Adamski J, Pischon T and Boeing H: Variation of serum metabolites related to habitual diet: a targeted metabolomic approach in EPIC-Potsdam. Eur J Clin Nutr 67: 1100-1108, 2013.

39. O'Keefe SJD, Li JV, Lahti L, Ou J, Carbonero F, Mohammed K, Posma JM, Kinross J, Wahl E, Ruder E, et al: Fat, fibre and cancer risk in African Americans and rural Africans. Nat Commun 6: 6342,2015 